\title{
Особенности мобильной системы WINDOWS 10 MOBILE
}

\author{
Садыков А.С., студент, \\ Технический институт (филиал) \\ Северо-Восточного федерального университета, \\ 2. Нерюнгри \\ E-mail: danzelnurka@mail.ru
}

Научный руководитель: к.n.н., зав. кафедрой МиИ Самохина В.М.

Мобильные приложения в жизни современного человека играют огромную роль, использование смартфона даёт пользователю возможность бесперебойно и автономно выполнять работу в соответствии со своими потребностями. Одна из наиболее оптимальных мобильных систем для работы с документами является Windows 10 Mobile. Наличие встроенного пакета Office и интеграция с сервисами Microsoft делают ее универсальной.

Пользователь выбирающий смартфон с данной системой, опирается на стабильность и плавность системы. Даже на низко производительном процессоре она бесперебойно и быстро выполняет свои функции. Система имеет оконный интерфейс, с постоянно обновляющей информации на иконках в зависимости от приложения, с возможностью редактирования, добавления на начальный экран нужных программ. (рис.1)
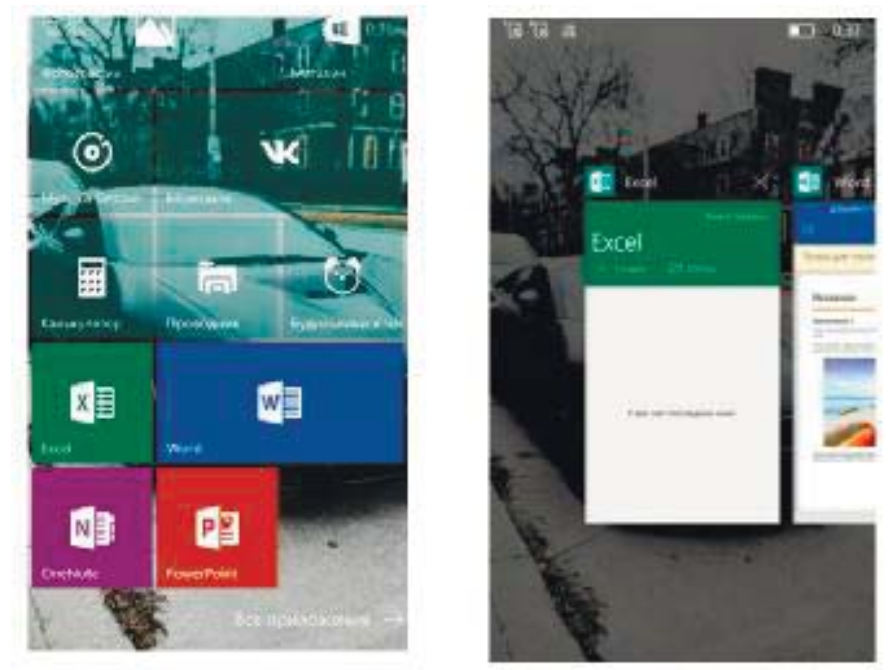

Рис. 1. Начальный экран

По сравнении с Android выбор смартфонов на данной системе не велик. Производителями являются Nokia и Microsoft. Основные возможности, которые предоставляют смартфоны на Windows 10 Mobile является высокопроизводительная работа система, синхронизация со встроенными сервисами Microsoft, работа с пакетом программ Office, которые уже установлены по умолчанию.

Создавая документ на смартфоне, имеется возможность сохранить его на облачное хранилище файлов OneDrive, и получить доступ с любого устройства и продолжить работу на персональных компьютерах или ноутбуках. Для этого требуется регистрация аккаунта Microsoft. (рис.2) 


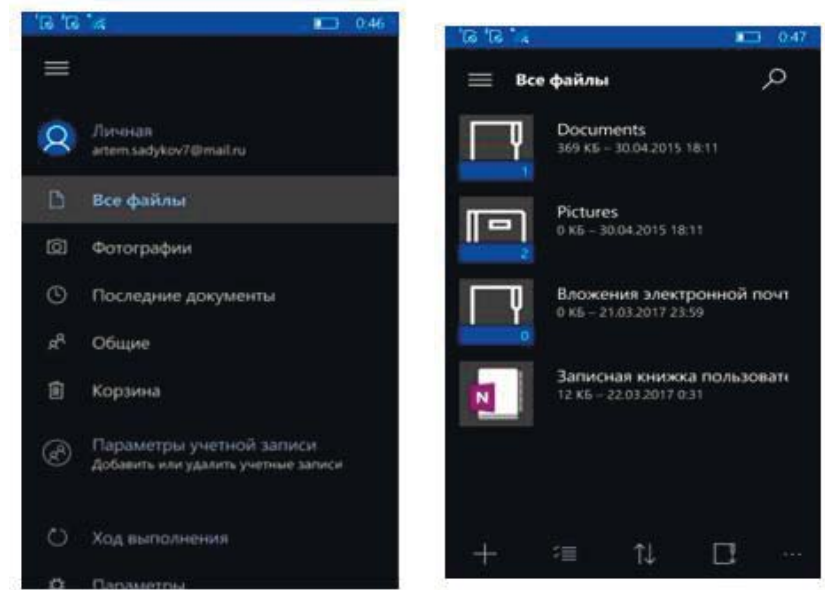

Рис. 2. Облачное хранилище OneDrive

Ещё одним достоинством системы является интуитивно понятный интерфейс, все настройки открытые, сгруппированы.

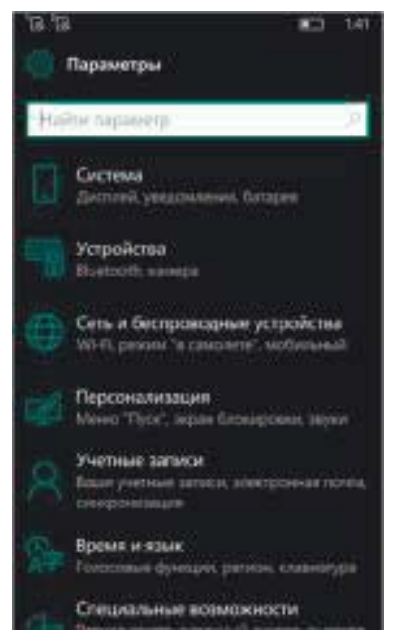

Рис. 3. Параметры

B отличие от IOS система предоставляет работу с файлами, имеется проводник, но в то же время она остается закрытой, и благодаря этому отсутствуют вирусы (рис.4):
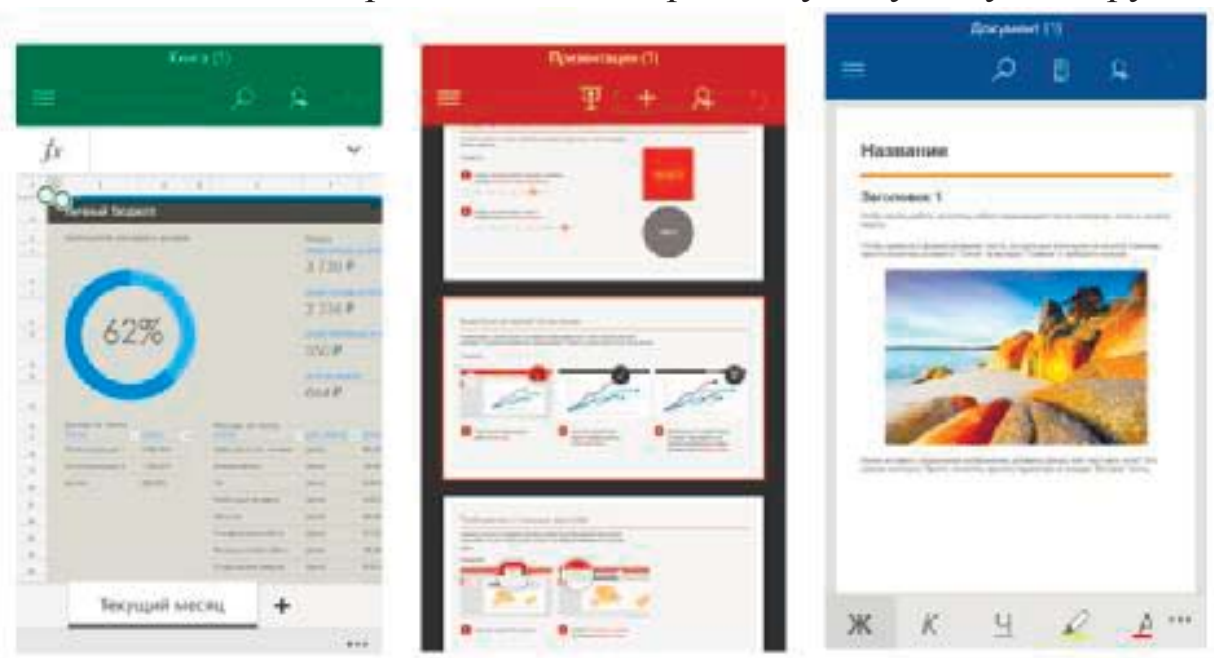

Рис. 4. Работа с пакетом Office

Помимо этого предусмотрен браузер Microsoft Edge и карты с навигацией (рис. 5): 


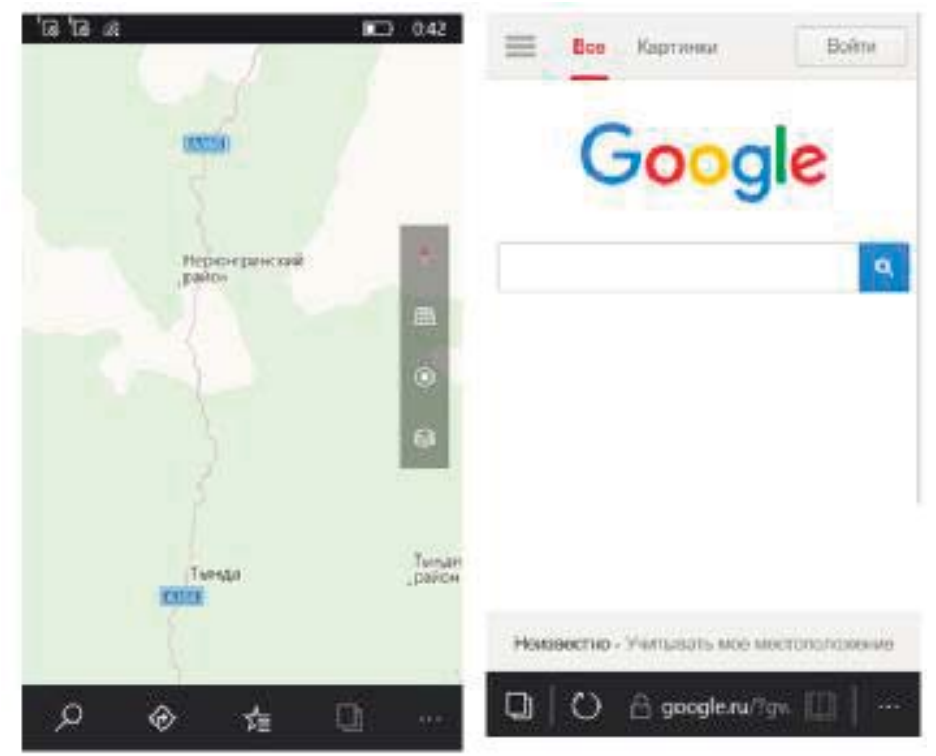

Рис. 5. Браузер и навигация

Имеются различные приложения, значительно расширяющие функционал системы, для этого имеется встроенный магазин. Все основные приложения, существующие на других ОС, существуют и здесь. Но есть и нехватка приложений, а некоторые могут работать с ошибками. Так как система занимает 3-4\% рынка мобильных устройств количество разработчиков приложений снижается (рис.6):

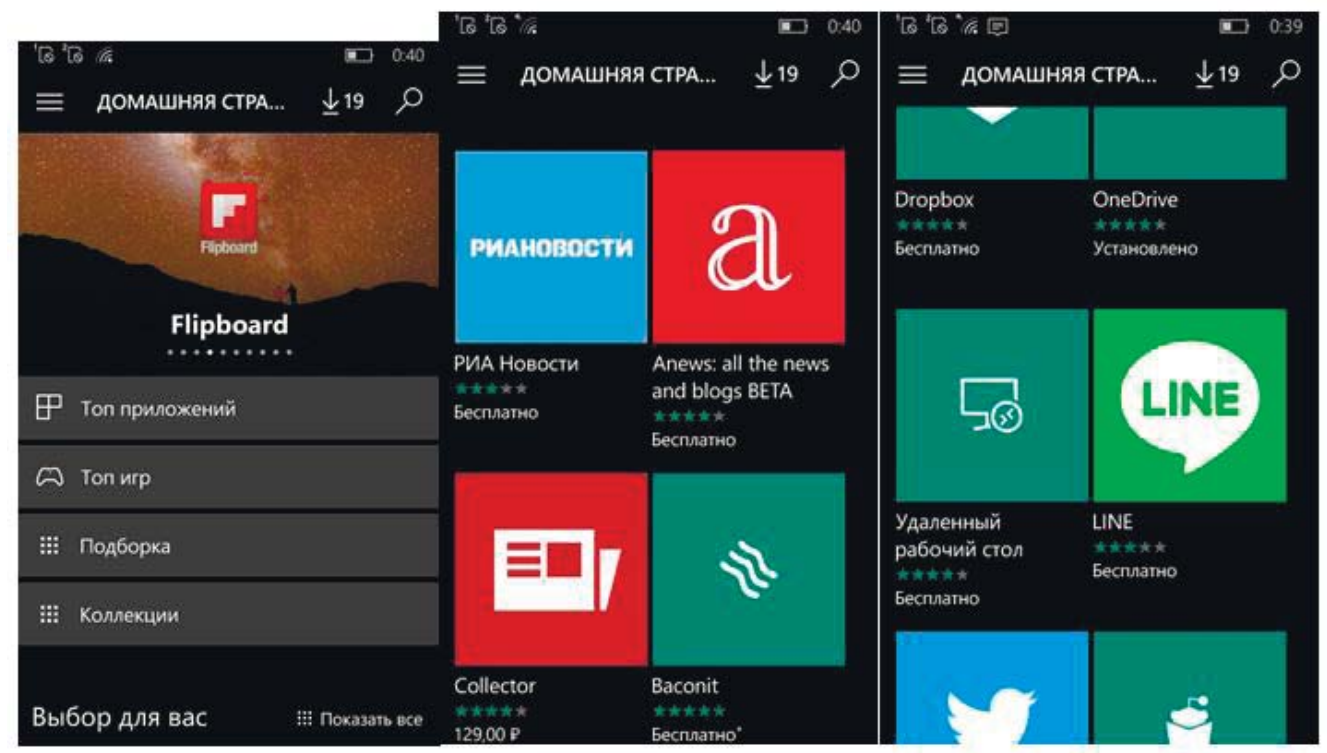

Рис. 6. Магазин приложений Windows Phone

Используя функцию Continium, можно превратить смартфон в переносной компьютер, подключив к нему мышь либо клавиатуру. Для этого требуется докстанция. На мониторе ПК будет изображен полноценный рабочий стол мобильной системы Windows 10 Mobile.

Для данной системы есть возможность создания приложений. Основным инструментом является Visual Studio. Интегрированные средства разработки предоставляют разработчику полноценные возможности отладки на устройстве и эмуляторе, такие же, как у разработчиков приложений под настольную версию Windows [2]. 
Expression Blend - это интерактивный визуальный дизайнер для XAML, технологии описания интерфейса для приложений Silverlight и Windows Presentation Foundation (WPF). Это отличное средство разработки, которое позволяет просто манипулировать слоями, анимацией, стилями и шаблонами. Это базовое средство разработки на XAML, программа Expression Blend не бесплатна, однако специальная версия для создания дизайнов приложений под Windows Phone под названием Expression Blend 4 for Windows Phone доступна для разработчиков бесплатно. [1]

Windows Phone Emulator предоставляет мощную среду, позволяющую практически полностью разработать приложение в эмуляторе.

Эмулятор поставляется только с одним встроенным приложением Internet Explorer, но это Internet Explorer 9 с поддержкой HTML5.

При этом эмулятор позволяет тестировать звонки и отсылку SMS-сообщений, поддерживает мультитач на мониторах с его поддержкой, поддерживает симуляцию камеры, геолокационных сервисов и акселерометра, а также позволят делать снимки экрана.

\section{Windows Phone Developer Registration Tool}

Перед тем, как разработчик сможет развернуть свое приложение на реальном устройстве, его необходимо зарегистрировать как устройство разработчика. Это делается один раз для определенного телефона. Зарегистрированный на Marketplace разработчик может зарегистрировать до 3 устройств (для разработчика, зарегистрированного как студент количество устройств ограничено до одного).

Silverlight Toolkit for Windows Phone- набор полезных элементов управления Silverlight для Windows Phone c поддержкой режима дизайна, от команды разработчиков Silverlight.[2]

В ходе написания научной статьи была рассмотрена мобильная операционная система Windows 10 Mobile.

Система является самой молодой, несмотря на малое количество приложений она имеет и свои плюсы. Это неповторимый дизайн, интеграция с сервисами Microsoft, топовые камеры от производителей, более лучшая работа с аккумулятором устройства, оптимизированная работа. Система заинтересует, прежде всего, людей, полностью погруженных в работу, так как включает пакет офисных программ, и возможность подключения телефона и превращение его в переносной компьютер. Данная система находиться ещё на стадии закрепления, на рынке. Нужна большая команда энтузиастов, которая поможет ей вырваться вперед. Возможность программирования приложения для неё, создает большие перспективы для её использования.

\section{Список литературы:}

1. Николаи М. Джосаттис (Nicolai M. Josuttis) - Стандартная библиотека C++: справочное руководство, 2-е издание, 2014 (The C++ Standard Library - A Tutorial and Reference, 2nd Edition, 2012)

2. Тихомиров В.А. Т462 Разработка простейших приложений для мобильных устройств: учеб. пособие. - Комсомольск-на-Амуре: ФГБОУ ВПО «КнаГТУ», 2013134c. 